\title{
An ER translocon for multi-pass membrane protein biogenesis
}

\section{Philip T McGilvray ${ }^{1}$, S Andrei Anghel ${ }^{1}$, Arunkumar Sundaram ${ }^{1}$, Frank Zhong ${ }^{1}$, Michael J Trnka ${ }^{2}$, James R Fuller ${ }^{1}$, Hong Hu${ }^{1}$, Alma L Burlingame ${ }^{2}$, Robert J Keenan ${ }^{1}$}

\author{
${ }^{1}$ University of Chicago, Chicago, United States of America;
}

${ }^{2}$ University of California, San Francisco, United States of America

\section{bkeenan@uchicago.edu}

Membrane proteins with multiple transmembrane domains play critical roles in cell physiology, but little is known about the machinery coordinating their biogenesis at the endoplasmic reticulum. Here we describe a $\sim 360 \mathrm{kDa}$ ribosome-associated complex comprising the core Sec61 channel and five accessory factors: TMCO1, CCDC47 and the Nicalin-TMEM147-NOMO complex. Cryoelectron microscopy reveals a large assembly at the ribosome exit tunnel organized around a central membrane cavity. Similar to protein-conducting channels that facilitate movement of transmembrane segments, cytosolic and luminal funnels in TMCO1 and TMEM147, respectively, suggest routes into the central membrane cavity. High-throughput mRNA sequencing shows selective translocon engagement with hundreds of different multi-pass membrane proteins. Consistent with a role in multi-pass membrane protein biogenesis, cells lacking different accessory components show reduced levels of one such client, the glutamate transporter EAAT1. These results identify a new human translocon and provide a molecular framework for understanding its role in multi-pass membrane protein biogenesis.

Keywords: membrane protein biogenesis, cryo-EM, translocon, multi-pass, TMCO1 